\title{
Unexpected Stability of Lentivirus upon Freeze-Thaw Cycles
}

Shaoshuai Meng M.Phil.

School of Life Sciences, Peking University

Biomedical Pioneering Innovation Center, Peking University

mss@pku.edu.cn

Wanting Yu Ph.D.

School of Life Sciences, Peking University

Biomedical Pioneering Innovation Center, Peking University

\section{Dear Editor,}

Characterizing virus stability at real-world conditions is crucial for infectious disease mitigation ${ }^{1,2}$. It is widely believed that virus are severely affected by repeated freeze-thaw cycles ${ }^{3,4}$. However, no studies have been conducted, to our knowledge, directly detailing this issue. Here, we present the effects on the infectivity of lentivirus upon multiple rounds of freeze-thaw cycles at different conditions. Surprisingly, we found that lentivirus is quite stable at freeze-thaw stress. It was able to keep up to about $40 \%$ activity after 10 freeze-thaw cycles.

We directly used GFP-expressing lentivirus for testing of its freeze-thaw stability. Briefly, clarified lentivirus containing DMEM supernatants was added to 293T cells cultured in 12-well plates (3.5x $10^{5}$ cells per well) at $5 \mu$ l per well directly or after $1,3,5,10$ freeze-thaw cycle(s) and GFP positive rate of transduced cells was measured afterwards. As for the freeze-thaw cycles, $30 \mu$ lentivirus in EP tubes (Thermo Scientific ${ }^{\mathrm{TM}}$ 90410; $1.5 \mathrm{~mL}$, low protein binding) was fast freezon in liquid nitrogen and then thawed on ice (completely thawed after about 40 mins) or in $37{ }^{\circ} \mathrm{C}$ water bath (completely thawed after about $1 \mathrm{~min}$ ). After refreshing medium about 18 hours later, GFP positive rate was measured by flow cytometry (BD FACS Aria III) 40 hours post transduction.

We found that the decay rates of lentivirus infectivity were similar between quick $\left(37^{\circ} \mathrm{C}\right.$ water bath) and slow (on ice) thawing conditions. About $83.5 \%(95 \% \mathrm{Cl}: 69.2 \%-97.9 \%), 67.0 \%(95 \% \mathrm{Cl}$ : 59.9\%-74.1\%), 51.2\% (95\%Cl: 45.1\%-57.3\%), 38.4\% (95\%Cl: 31.0\%-45.8\%) infectivity was kept at quick thaw condition and $83.2 \%(95 \% \mathrm{Cl}$ : 51.6\%-114.9\%), 54.4\% (95\% Cl: $45.6 \%-63.1 \%), 48.3 \%$ (95\%Cl: $38.6 \%-58.1 \%), 40.1 \%$ (95\%Cl: $16.5 \%-63.8 \%)$ infectivity was kept at slow thaw condition after 1, 3, 5, 10 freeze-thaw cycles, respectively (Fig. 1).

We acknowledge that it only represents lentivirus in culture medium supernatants. Nevertheless, our data demonstrated that virus could be quite resistant to freeze-thaw cycles. Our finding strongly suggests that life threatening high-impact virus should be further characterized for its 
stability at real-world conditions. Particularly, facing this pandemic challenge, we should carefully evaluate the possibility of transmission of virus through cold chain etc. And stringent usage of personal protective equipment and intensify virus surveillance should be implanted for cold chain related workers.

\section{Contributors}

SM conceived the study. SM designed the study and performed the experiments. SM and WY analyzed the data. SM wrote the manuscript. All authors approved the final version of the manuscript.

\section{Declaration of interests}

All authors declare no competing interests. This study did not receive any external financial supports.

\section{Acknowledgments}

The authors thank Prof. Yujie Sun of Peking University for helping revising \& editing the manuscript; the staffs of high throughput facility of Biomedical Pioneering Innovation Center (BIOPIC), Peking University for helping performing flow cytometry experiments.

\section{REFERENCES}

1. van Doremalen, N. et al. Aerosol and Surface Stability of SARS-CoV-2 as Compared with SARS-CoV-1. N. Engl. J. Med. NEJMc2004973 (2020). doi:10.1056/NEJMc2004973

2. Lee, J. R. et al. Modeling the Stability of Severe Acute Respiratory Syndrome Coronavirus 2 (SARS-CoV-2) on Skin, Currency, and Clothing. medRxiv (2020). doi:10.11693/hyhz20181000233

3. Care and Handling of Viruses | Wu Tsai Neurosciences Institute. Available at: https://neuroscience.stanford.edu/research/programs/community-labs/neurosciencegene-vector-and-virus-core/care-and-handling-viruses. (Accessed: 6th August 2020)

4. Tiscornia, G., Singer, O. \& Verma, I. M. Production and purification of lentiviral vectors. Nat. Protoc. 1, 241-245 (2006).

Fig. 1: Lentivirus remains stable upon multiple rounds of freeze-thaw cycles at $37^{\circ} \mathrm{C}$ water bath or ice thaw conditions. (A) Typical flow cytometry data. (B) Statistical data of 3 biological replicates. 
A

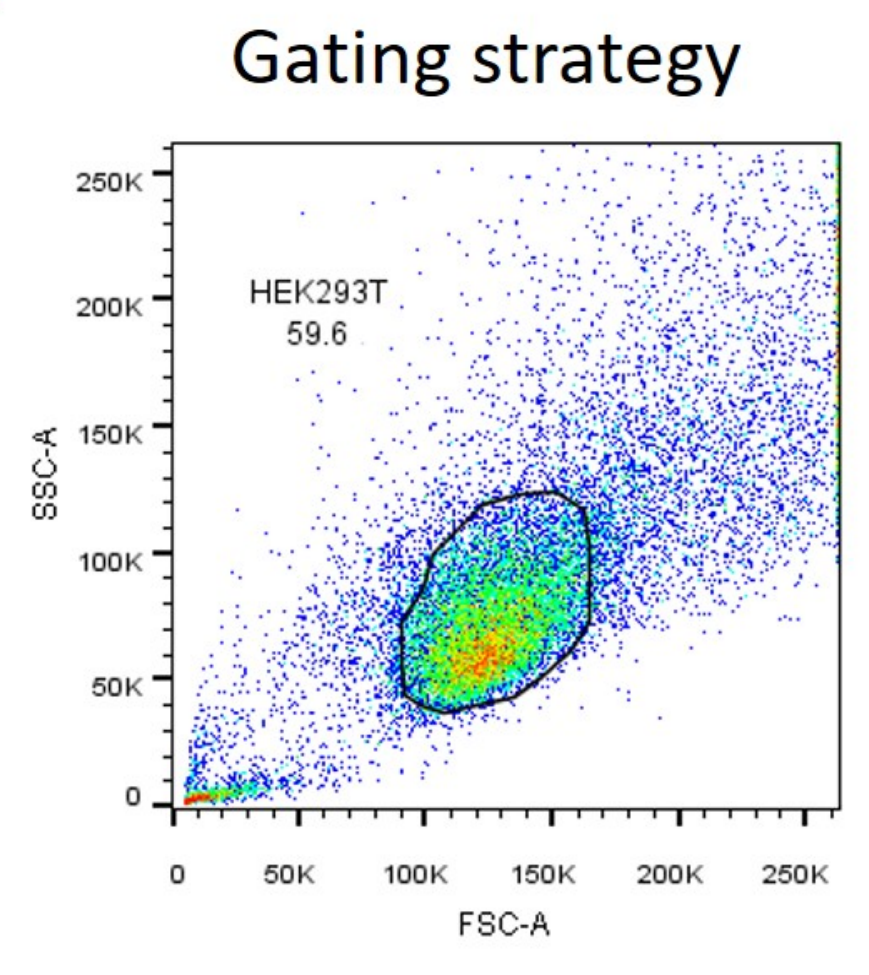

Non transduction control

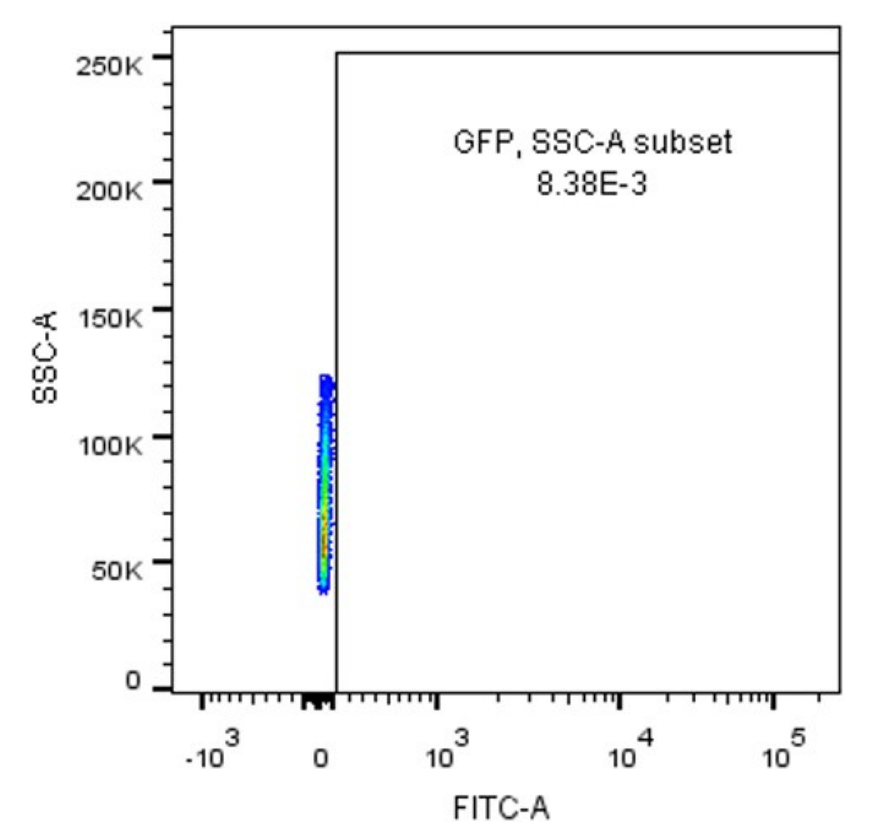

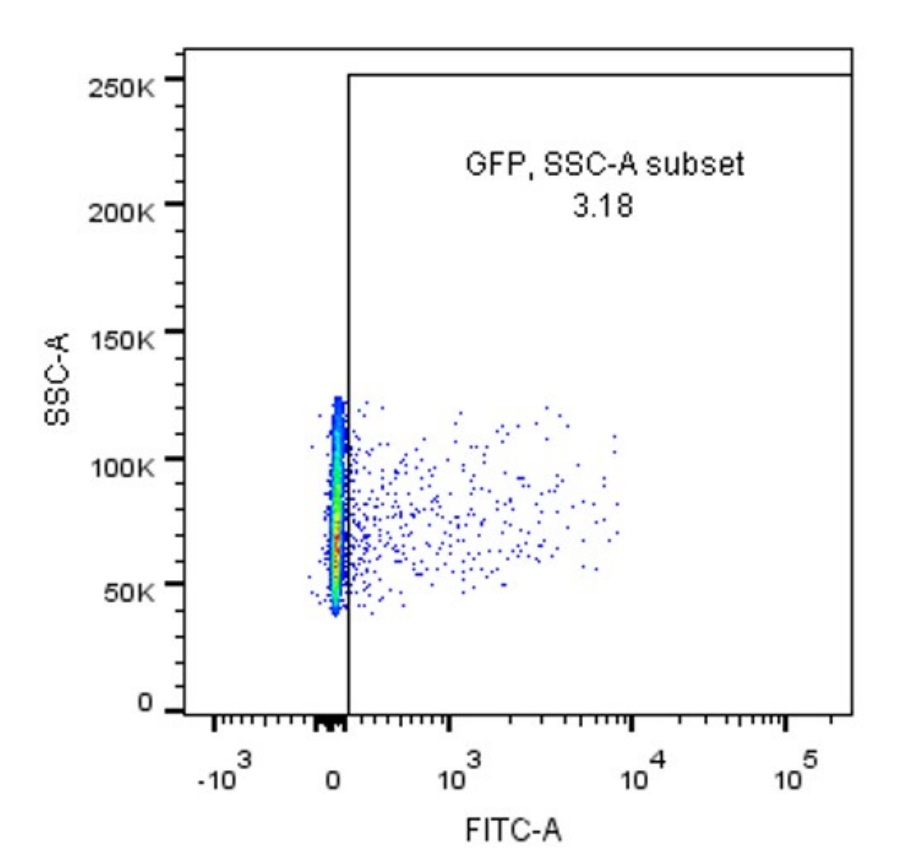
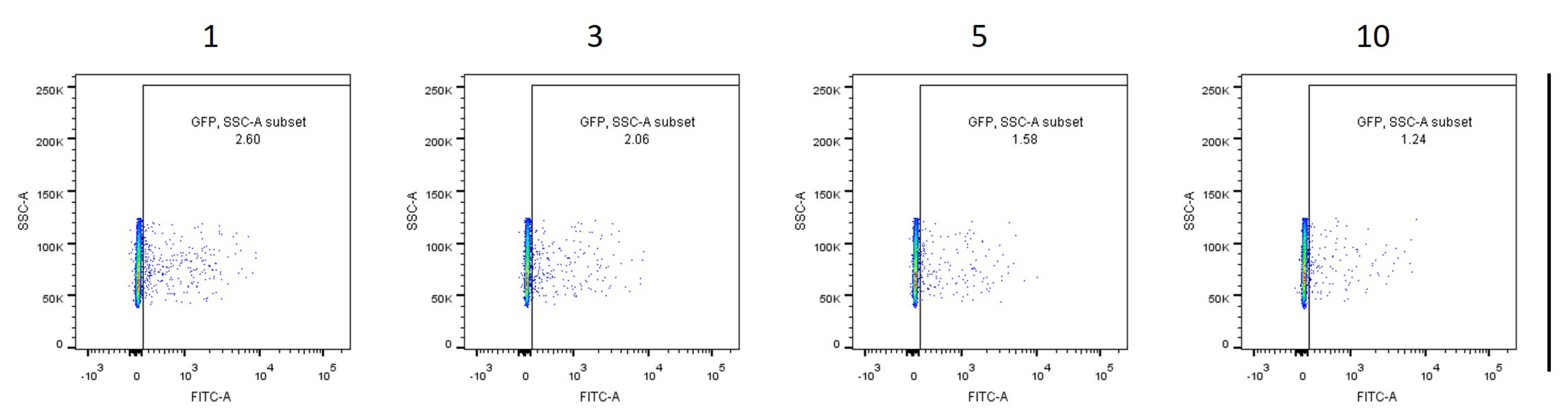

$37^{\circ} \mathrm{C}$

water

bath

B
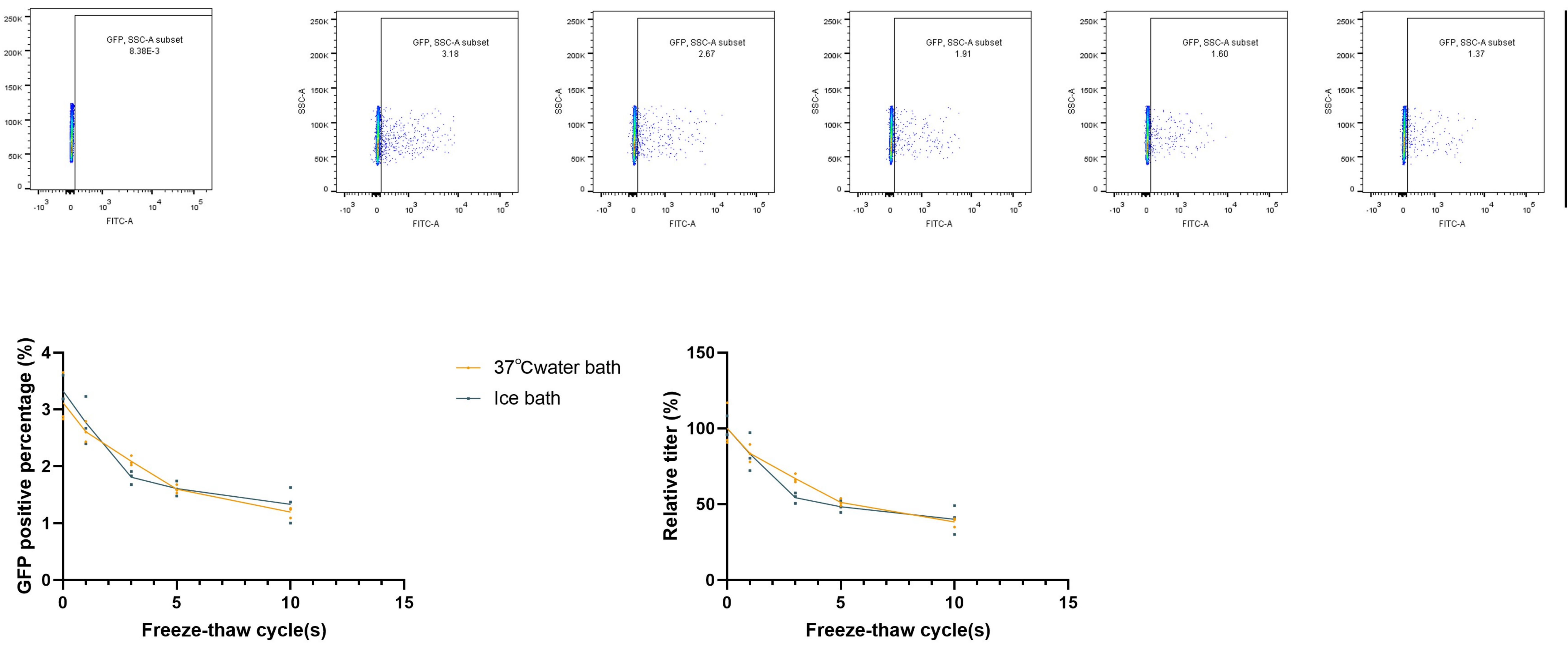

$-37^{\circ} \mathrm{Cwater}$ bath

- Ice bath

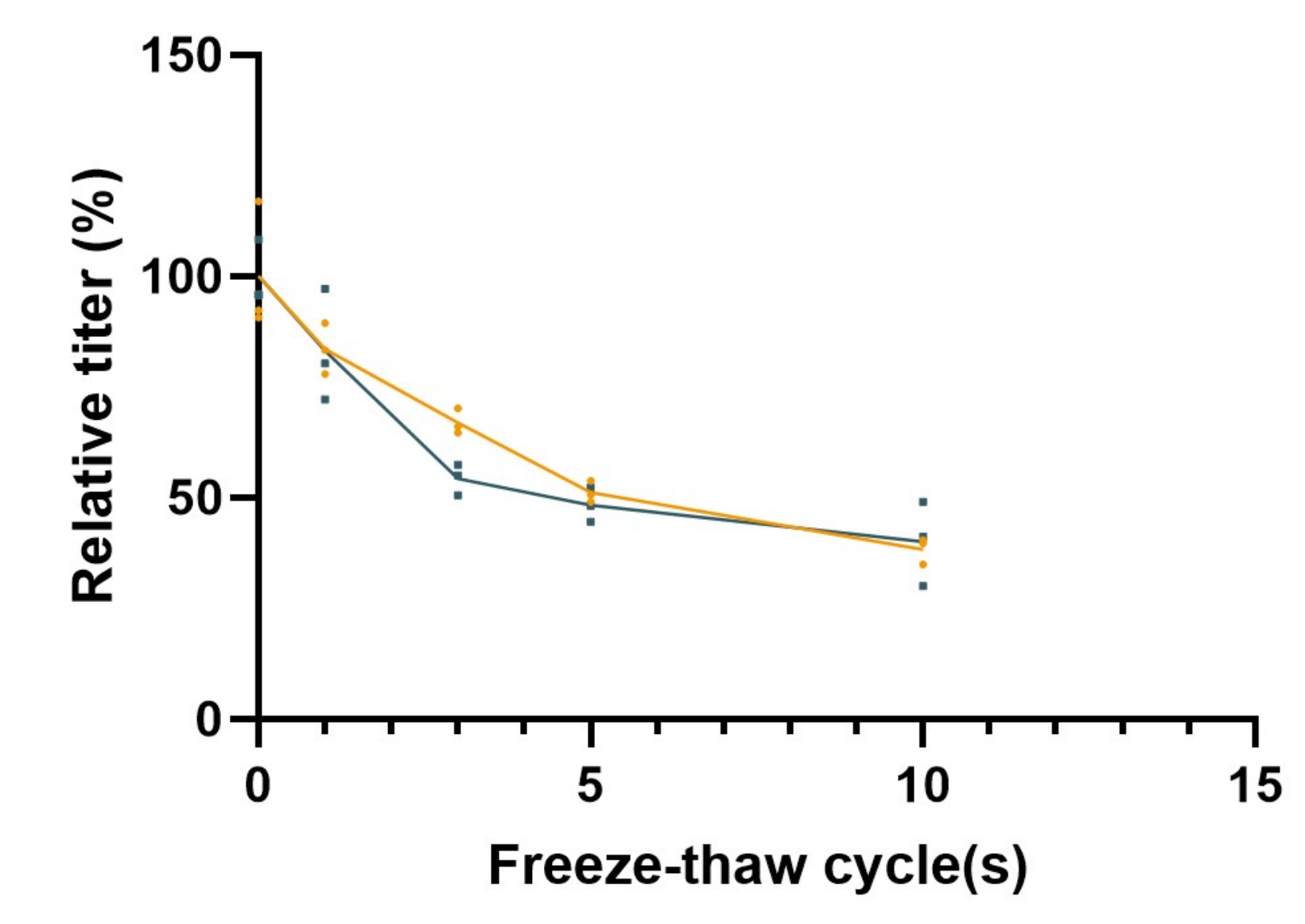

